\title{
DISCUSSION
}

\section{Overactive bladder: paediatric aspects}

\author{
D.A. BLOOM \\ Division of Urology, University of Michigan Medical Center, Department of Paediatric Surgery, Ann Arbor, Michigan, USA
}

\section{Introduction}

Like Dr Nijman, I see many children with an overactive bladder and face similar dilemmas trying to understand and manage the variable signs and symptoms in the absence of a unifying pathophysiological model. This symposium has brought together a diverse group of clinicians and scientists in search of such a model, and I particularly enjoyed Dr Nijman's analysis of the paediatric aspects.

\section{Complexity has its price}

Dr Nijman said that lower urinary tract function, which he called 'control', is a complex process of nervous system development and behavioural modification; indeed it is. This complexity is part of the price humans pay for their phylogenetic position, compared with, for example. freshwater coelenterates, which have only a single stoma for entry and exit of nutrients. Higher evolutionary life-forms, of necessity, evolved more sophisticated systems to manage elimination functions. For mammals, elimination is more than species-specific; in humans, various stages of life, lifestyles and transient situations place special demands on their excretory function. In fact, the fetus and infant control their lower urinary tracts very effectively, just as they control their puImonary, cardiovascular and gastrointestinal systems. The difficulties lie in the assumption of conscious control over a previously neuro-viscerally controlled system, because of the effects of the elements of consciousness, behaviour, personality and social pressures. Most people manage these hurdles effectively, but it should come as no surprise that many children have some difficulty attaining cognitive management of the lower urinary tract. Most children surmount their problems, but for a few children the problems we label as dysfunctions become a way of life. There is probably one fairly simple unifying pathophysiological explanation for these dysfunctions.

\section{Common terminology of 'eliminology'}

Efforts to understand the lower urinary tract so that these dysfunctions can be treated brings me to the Sapir-Whorf hypothesis [1]. This illuminating linguistic tenet states that 'the language we speak shapes the world we see.' That is, our ability to understand and define the reality in which we live is determined by the language we have at hand. The topic of this symposium is the overactive bladder, a term relatively free of causal implications and vested interests, thereby leaving some freedom for discussion and hypothesis. Indeed, terminology is changing; today there is a preference for the terms 'unstable bladder' and 'detrusor hyper-reflexia' and we are in the process of discarding archaic terms. Dr Nijman uses the term 'functional incontinence' for the common daytime accidents that have no neuroanatomical basis, i.e. none that have been identified thus far. Happily, as we have seen during this symposium, there seems to be emerging agreement on most of the terms in our 'eliminology' lexicon.

\section{Models help to comprehend phenomena}

Intellectual tools have been developed to understand and ultimately control nature, by reducing bits of the cosmos to finite components and analysing them in terms of models. This is a unique human skill and Delbanco's description of this skill transcends his literary intent: '...individual human beings can break free of the structures of thought into which they are born and, by re-imagining the world, they can change it.' [2]. The present model of the lower urinary tract has grown from a static set of tubes and reservoir to a marvel of bioegineering still beyond our complete grasp. There is a continuing need to re-imagine it by making new models and testing them. Any new models must be pruned with the parsimonious razor of the ancient English monk William of Ockham, who said 'entia non sunt multiplicanda praeter necessitate', which means that the simplest hypothesis is usually the best. There should be caution about accepting models that seem too complex to understand. Our linguistic freedom and our skill with 
models might allow us to consider the overactive bladder as a disorder of the pelvic floor or guarding reflex dysfunction. Rather than blaming the bladder primarily for this disorder, perhaps it is possible that the 'on/off switch' is faulty [3].

\section{Classification helps rationalize therapy}

Dr Nijman's classification of bladder dysfunction is functional and urodynamic. The problems could just as easily be classified aetiologically, symptomatically or therapeutically [4]. Yet however voiding dysfunctions are viewed, the real task is to treat them. Current therapy includes not only pharmacological tools, but also behavioural, biofeedback, electric and surgical options. Dr Nijman views the behavioural and biofeedback modalities as cognitive therapy. However, in the absence of a unifying pathophysiological model, therapy remains heuristic.

\section{Heuristics are operational judgements}

Heuristic decisions for therapy are made without the support of complete informative data. McDonald has written convincingly about the virtues and risks of medical heuristics [5]. The failure of a substantial subset of patients with lower urinary tract dysfunction to respond to therapy of any sort should come as no surprise, given the lack of a unified model for the dysfunctions and the heuristic nature of the therapies. Clearly, the data are far from complete. For example, we recognize the typical side-effects of commonly used agents, such as anticholinergics, as personality changes and schooling inattentiveness, but it is equally likely that deleterious subclinical effects may also occur. This could be an important issue in children during the critical early years of schooling and socialization. Such concerns offer fertile ground for some important clinical investigation.

\section{References}

1 Bloom D, Park J, Koo HP. Comments on pediatric elimination dysfunctions: the Whorf hypothesis, the elimination interview, the guarding reflex and nocturnal enuresis. Eur Urol 1998: 33: 20-4

2 Delbanco A. Required Reading, Why Our American Classics Matter Now. New York: Farrar, Straus and Giroux, 1997:9

3 Park J, Bloom DA, McGuire EJ. The guarding reflex revisited. Br J Urol 1997; 80: 940-5

4 Bloom DA, Faerber G, Bomalaski MD. Urinary incontinence in girls. Evaluation, treatment and its place in the standard model of voiding dysfunctions in children. Urol Clin North Am 1995; 22: 521-38

5 McDonald CJ. Medical heuristics; the silent adjudicators of clinical practice. Ann Int Med 1996; 124: 56-62

Correspondence: D.A. Bloom, MD, University of Michigan Medical Center, Department of Paediatric Surgery, $1500 \mathrm{E}$ Medical Center Drive. Room 2916, Ann Arbor, MI 48109-0718, USA. 